Pacific

Journal of

Mathematics

ON MIXED PRODUCTS OF COMPLEX CHARACTERS OF THE DOUBLE COVERS OF THE SYMMETRIC GROUPS

Christine Bessenrodt 


\title{
ON MIXED PRODUCTS OF COMPLEX CHARACTERS OF THE DOUBLE COVERS OF THE SYMMETRIC GROUPS
}

\author{
Christine Bessenrodt
}

\begin{abstract}
In this article, two families of (almost) homogeneous mixed Kronecker products of non-faithful and of spin characters of the double covers of the symmetric groups are described. This is then applied to classify the irreducible mixed products, thus completing the classification of all irreducible Kronecker products of characters of the double covers of the symmetric groups.
\end{abstract}

\section{Introduction.}

Kronecker products of complex characters of the symmetric group $S_{n}$ have been studied in many papers. Information on special products and on the coefficients of special constituents have been obtained but there is no efficient combinatorial algorithm in sight for computing these products. In [1], products of $S_{n}$-characters with few homogeneous components and homogeneous products of characters of the alternating group $A_{n}$ have been classified. In particular, there are no non-trivial homogeneous Kronecker products for $S_{n}$, but there are such products for $A_{n}$, when $n$ is a square number (these are even irreducible).

For the double covers $\widetilde{S}_{n}$ of the symmetric groups, information about products of characters is even more sparse. Recently, in [2] some results have been obtained on products of spin characters of $\widetilde{S}_{n}$ which led to a classification of homogeneous spin products. Here, homogeneous products do occur for all triangular numbers $n$, but non-trivial irreducible products occur only for $n=6$.

In this article, we consider mixed products of complex characters for the double covers $\widetilde{S}_{n}$, i.e., products of a non-faithful character of $\widetilde{S}_{n}$ (corresponding to a character of $S_{n}$ ) with a spin character. In this situation, there are some interesting homogeneous or almost homogeneous mixed products; finding such mixed products was greatly helped by the special maple packages SF and QF for dealing with symmetric functions by John Stembridge. Two families of homogeneous resp. almost homogeneous products are described; one for any composite number and the other one for triangular numbers. The irreducible mixed products are then classified; they occur for even numbers and triangular numbers satisfying a congruence condition. 


\section{Preliminaries.}

We denote by $P(n)$ the set of partitions of $n$. For a partition $\lambda \in P(n)$, $l(\lambda)$ denotes its length, i.e., the number of (non-zero) parts of $\lambda$. The set of partitions of $n$ into odd parts only is denoted by $O(n)$, and the set of partitions of $n$ into distinct parts is denoted by $D(n)$. We write $D^{+}(n)$ resp. $D^{-}(n)$ for the sets of partitions $\lambda$ in $D(n)$ with $n-l(\lambda)$ even resp. odd; the partition $\lambda$ is then also called even resp. odd.

We write $S_{n}$ for the symmetric group on $n$ letters, and $\widetilde{S}_{n}$ for one of its double covers; so $\widetilde{S}_{n}$ is a non-split extension of $S_{n}$ by a central subgroup $\langle z\rangle$ of order 2. It is well-known that the representation theory of these double covers is 'the same' for all representation theoretical purposes. The spin characters of $\widetilde{S}_{n}$ are those that do not have $z$ in their kernel. For an introduction to the properties of spin characters resp. for some results we will need in the sequel we refer to $[\mathbf{5}],[\mathbf{1 0}],[\mathbf{1 1}],[\mathbf{1 3}]$. Below we collect some of the necessary notation and some results from $[\mathbf{1 3}]$ that are crucial in later sections.

For $\lambda \in P(n)$, we write $[\lambda]$ for the corresponding irreducible character of $S_{n}$; this is identified with the corresponding character of $\widetilde{S}_{n}$. The associate classes of spin characters of $\widetilde{S}_{n}$ are labelled canonically by the partitions in $D(n)$. For each $\lambda \in D^{+}(n)$ there is a self-associate spin character $\langle\lambda\rangle=$ $\operatorname{sgn}\langle\lambda\rangle$, and for each $\lambda \in D^{-}(n)$ there is a pair of associate spin characters $\langle\lambda\rangle,\langle\lambda\rangle^{\prime}=\operatorname{sgn}\langle\lambda\rangle$. We write

$$
\begin{aligned}
& \widehat{\langle\lambda\rangle}= \begin{cases}\langle\lambda\rangle & \text { if } \lambda \in D^{+}(n) \\
\langle\lambda\rangle+\langle\lambda\rangle^{\prime} & \text { if } \lambda \in D^{-}(n)\end{cases} \\
& \varepsilon_{\lambda}= \begin{cases}1 & \text { if } \lambda \in D^{+}(n) \\
\sqrt{2} & \text { if } \lambda \in D^{-}(n) .\end{cases}
\end{aligned}
$$

In [13], Stembridge introduces a projective analogue of the outer tensor product, called the reduced Clifford product, and proves a shifted analogue of the Littlewood-Richardson rule which we will need in the sequel. To state this, we first have to define some further combinatorial notions.

Let $A^{\prime}$ be the ordered alphabet $\left\{1^{\prime}<1<2^{\prime}<2<\ldots\right\}$. The letters $1^{\prime}, 2^{\prime}, \ldots$ are said to be marked, the others are unmarked. The notation $|a|$ refers to the unmarked version of a letter $a$ in $A^{\prime}$. To a partition $\lambda \in D(n)$ we associate a shifted diagram

$$
Y^{\prime}(\lambda)=\left\{(i, j) \in \mathbb{N}^{2} \mid 1 \leq i \leq l(\lambda), i \leq j \leq \lambda_{i}+i-1\right\} .
$$

A shifted tableau $T$ of shape $\lambda$ is a map $T: Y^{\prime}(\lambda) \rightarrow A^{\prime}$ such that $T(i, j) \leq$ $T(i+1, j), T(i, j) \leq T(i, j+1)$ for all $i, j$, and every $k \in\{1,2, \ldots\}$ appears at most once in each column of $T$, and every $k^{\prime} \in\left\{1^{\prime}, 2^{\prime}, \ldots\right\}$ appears at most once in each row of $T$. For $k \in\{1,2, \ldots\}$, let $c_{k}$ be the number of boxes 
$(i, j)$ in $Y^{\prime}(\lambda)$ such that $|T(i, j)|=k$ : Then we say that the tableau $T$ has content $\left(c_{1}, c_{2}, \ldots\right)$. Analogously, we define skew shifted diagrams and skew shifted tableaux of skew shape $\lambda \backslash \mu$ if $\mu$ is a partition with $Y^{\prime}(\mu) \subseteq Y^{\prime}(\lambda)$. For a (possibly skew) shifted tableau $S$ we define its associated word $w(S)=$ $w_{1} w_{2} \cdots$ by reading the rows of $S$ from left to right and from bottom to top. By erasing the marks of $w$, we obtain the word $|w|$.

Given a word $w=w_{1} w_{2} \ldots$, we define

$$
m_{i}(j)=\text { multiplicity of } i \text { among } w_{n-j+1}, \ldots, w_{n}, \quad \text { for } 0 \leq j \leq n
$$

$m_{i}(n+j)=m_{i}(n)+$ multiplicity of $i^{\prime}$ among $w_{1}, \ldots, w_{j}, \quad$ for $0<j \leq n$.

This function $m_{i}$ corresponds to reading the rows of the tableau first from right to left and from top to bottom, counting the letter $i$ on the way, and then reading from bottom to top and left to right, counting the letter $i^{\prime}$ on this way.

The word $w$ satisfies the lattice property if, whenever $m_{i}(j)=m_{i-1}(j)$, then

$$
\begin{array}{rlrl}
w_{n-j} & \neq i, i^{\prime}, & & \text { if } 0 \leq j<n \\
w_{j-n+1} & \neq i-1, i^{\prime}, & \text { if } n \leq j<2 n .
\end{array}
$$

For two partitions $\mu$ and $\nu$ we denote by $\mu \cup \nu$ the partition which has as its parts all the parts of $\mu$ and $\nu$ together.

Theorem 2.1 ([13,8.1 and 8.3]). Let $\mu \in D(k), \nu \in D(n-k), \lambda \in D(n)$, and form the reduced Clifford product $\langle\mu\rangle \times_{c}\langle\nu\rangle$. Then we have

$$
\left(\left(\langle\mu\rangle \times_{c}\langle\nu\rangle\right) \uparrow^{\widetilde{S}_{n}},\langle\lambda\rangle\right)=\frac{1}{\varepsilon_{\lambda} \varepsilon_{\mu \cup \nu}} 2^{(l(\mu)+l(\nu)-l(\lambda)) / 2} f_{\mu \nu}^{\lambda},
$$

unless $\lambda$ is odd and $\lambda=\mu \cup \nu$. In that latter case, the multiplicity of $\langle\lambda\rangle$ is 0 or 1 , according to the choice of associates.

The coefficient $f_{\mu \nu}^{\lambda}$ is the number of shifted tableaux $S$ of shape $\lambda \backslash \mu$ and content $\nu$ such that the tableau word $w=w(S)$ satisfies the lattice property and the leftmost $i$ of $|w|$ is unmarked in $w$ for $1 \leq i \leq l(\nu)$.

We will also use the following result from $[\mathbf{1 3}]$ on inner tensor products with the basic spin character $\langle n\rangle$ :

Theorem $2.2([\mathbf{1 3}, 9.3])$. Let $\lambda \in D(n), \mu$ a partition of $n$. We have

$$
(\langle n\rangle[\mu],\langle\lambda\rangle)=\frac{1}{\varepsilon_{\lambda} \varepsilon_{(n)}} 2^{(l(\lambda)-1) / 2} g_{\lambda \mu},
$$

unless $\lambda=(n), n$ is even, and $\mu$ is a hook partition. In that case, the multiplicity of $\langle\lambda\rangle$ is 0 or 1 according to choice of associates.

The coefficient $g_{\lambda \mu}$ is the number of "shifted tableaux" $S$ of unshifted shape $\mu$ and content $\lambda$ such that the tableau word $w=w(S)$ satisfies the lattice property and the leftmost $i$ of $|w|$ is unmarked in $w$ for $1 \leq i \leq l(\lambda)$.

As an interesting consequence, this implies 
Corollary 2.3. Let $n \in \mathbb{N}$. Then

$$
\langle n\rangle \cdot \widehat{\langle n\rangle}=[n]+\sum_{i=1}^{n-1}\left[n-i, 1^{i}\right] .
$$

Finally, we collect some information about certain constituents in squares resp. in 'almost' squares.

Theorem $2.4([\mathbf{1 2}],[\mathbf{1 4}],[\mathbf{1 5}])$. Let $n \in \mathbb{N}$. Let $\lambda \in P(n)$ with $\lambda \neq(n)$, $\left(1^{n}\right)$. Define $a, b \in \mathbb{N}_{0}$ by

$$
[\lambda]^{2}=[n]+a[n-2,2]+b\left[n-3,1^{3}\right]+\text { other constituents. }
$$

Denote by $h_{i}$ the number of hooks of $\lambda$ of length $i$, for $i \in\{1,2\}$. Let $h_{21}$ be the number of hooks of $\lambda$ of length 3 and arm length 1 . Then we have:

(i) $a=h_{2}+h_{1}\left(h_{1}-2\right)$.

In particular, $a>0$ if $n \geq 4$.

(ii) $b=h_{1}\left(h_{1}-1\right)\left(h_{1}-3\right)+\left(h_{1}-1\right)\left(h_{2}+1\right)+h_{21}$.

In particular, $b>0$, unless $\lambda$ is $(n-1,1)$ or $(n-1,1)^{\prime}$.

Theorem 2.5 ([8, Theorem 4.3]). Let $n \in \mathbb{N}, n \geq 4$. Let $\mu \in D(n)$ with $\mu \neq(n)$ and $\mu \neq(k, k-1, \ldots, 2,1)$. Let $s, t \in \mathbb{N}$ be defined by

$$
\langle\mu\rangle \cdot \overline{\langle\mu\rangle}=[n]+s[n-1,1]+t[n-2,2]+\text { other constituents. }
$$

Then:

(i) $t \geq 1$.

(ii) If $n \geq 5$, $\mu$ is even and $\mu$ is not of the form $(k+r, k-1+r, \ldots, 1+r)$ for some $r$, then $t \geq 2$.

(iii) If $\mu$ is not of the form $(k+r, k-1+r, \ldots, 1+r)$ for some $r$, then $s \geq 1$.

We have already considered the exceptional case $\mu=(n)$ in Corollary 2.3. Note that for even $n$ it is not clear which hook characters appear in the product $\langle n\rangle \cdot\langle n\rangle$ and which appear in the product $\langle n\rangle \cdot\langle n\rangle^{\prime}$, except that each product contains one out of a pair of conjugate hook characters. Clearly, both products do not contain $[n-2,2]$ as a constituent, i.e., for the basic spin character we have $t=0$ in the notation of the Theorem above.

The exceptional case of a staircase partition $\mu=(k, k-1, \ldots, 1)$ will be treated in the next section in Theorem 3.5.

\section{Almost homogeneous mixed products.}

We now want to study the case of mixed products, i.e., products of the form $\langle\mu\rangle \cdot[\nu]$. Of course, if we know all the constituents in the case of products of spin characters then we also know all the coefficients in the case of mixed products, since

$$
(\langle\mu\rangle\langle\nu\rangle,[\lambda])=(\langle\mu\rangle[\lambda], \overline{\langle\nu\rangle})
$$


and $\overline{\langle\nu\rangle}=\langle\nu\rangle$ or $\langle\nu\rangle^{\prime}$, depending on $n-l(\nu) \bmod 4$. But it is not clear how to obtain a classification of the homogeneous mixed products from the results we know so far. In the following, we will describe some "combinatorially homogeneous" mixed products, and we classify the irreducible mixed products. We call a character of $\widetilde{S}_{n}$ "homogeneous" if it is of the form $c\langle\lambda\rangle$ resp. "almost homogeneous" if it is of the form $c \widehat{\langle\lambda\rangle}$ for some $\lambda \in D(n)$ and $c \in \mathbb{N}$.

We will need the following combinatorial result (see [2], Lemma 4.1 and its proof).

Lemma $3.1([2])$. Let $k \in \mathbb{N}$. Let $H(k)$ denote the product of the hook lengths in $\nu=(k, k-1, \ldots, 2,1)$, and let $B(k)$ denote the product of the bar lengths in $\nu$. Set $n=\frac{1}{2} k(k+1)$. Then:

(i) $B(k)=2^{n-k} H(k)$.

(ii) $B(k+1)=B(k) \prod_{j=1}^{k+1}(k+j)$.

First we want to classify all homogeneous resp. almost homogeneous products with the basic spin character $\langle n\rangle$.

Theorem 3.2. Let $\mu \in P(n), \mu \neq(n),\left(1^{n}\right)$. Then the product $\langle n\rangle \cdot[\mu]$ is almost homogeneous if and only if $\mu$ is a rectangle.

Up to conjugation, we may assume in this case that $\mu=\left(b^{a}\right)$ with $1<$ $a \leq b$, and then the product is

$$
\langle n\rangle \cdot\left[b^{a}\right]= \begin{cases}2^{\frac{a-3}{2}}\langle a+b-1, a+\widehat{b-3}, \cdots, b-a+1\rangle, & \text { if } a \text { is odd and } \\ 2^{\left[\frac{a-1}{2}\right]}\langle a+b-1, a+b-3, \cdots, b-a+1\rangle, & \text { else. }\end{cases}
$$

Proof. First assume that $\mu$ is not a rectangle. Let $h_{i i}, i=1, \ldots, d=d(\mu)$, denote the principal hook lengths in $\mu$. By Theorem 2.2 the product $\langle n\rangle[\mu]$ always has a constituent $\left\langle h_{11}, h_{22}, \ldots, h_{d d}\right\rangle$ as is illustrated by the following tableau for $\mu=\left(7^{3}, 6^{2}\right)$ :

$\begin{array}{lllllll}1^{\prime} & 1 & 1 & 1 & 1 & 1 & 1 \\ 1^{\prime} & 2^{\prime} & 2 & 2 & 2 & 2 & 2 \\ 1^{\prime} & 2^{\prime} & 3^{\prime} & 3 & 3 & 3 & 3 \\ 1^{\prime} & 2^{\prime} & 3^{\prime} & 4^{\prime} & 4 & 4 & \\ 1 & 2 & 3 & 4 & 5 & 5 & \end{array}$

If $\mu$ is not a rectangle, then take $j$ maximal with $\mu_{j}>\mu_{j+1}>0$. We can then replace the final two entries $j^{\prime} j$ in the $j$ th column by the entries $j j+1$ and still obtain a tableau of the type counted by the coefficients $g_{\lambda \mu}$ occurring in Theorem 2.2, giving a constituent labelled by a partition different from 
$\left(h_{11}, h_{22}, \ldots, h_{d d}\right)$. Thus if the product $\langle n\rangle \cdot[\mu]$ is almost homogeneous then $\mu$ has to be a rectangle.

So now we consider the case that $\mu$ is a rectangle, and we may assume that $\mu=\left(b^{a}\right)$ with $a \leq b$. In this situation, we have for the partition considered above:

$$
\lambda=\left(h_{11}, h_{22}, \ldots, h_{d d}\right)=(a+b-1, a+b-3, \ldots, b-a+1) .
$$

The multiplicity of the constituent $\langle a+b-1, a+b-3, \ldots, b-a+1\rangle$ in the product can be calculated by Theorem 2.2 ; it is easily seen that $g_{\lambda \mu}=1$, and hence the multiplicity is

$$
\frac{1}{\varepsilon_{\lambda} \varepsilon_{(n)}} 2^{\frac{a-1}{2}}= \begin{cases}2^{\frac{a-3}{2}}, & \text { if } a \text { is odd and } b \text { is even } \\ 2^{\left[\frac{a-1}{2}\right]}, & \text { else, }\end{cases}
$$

as is easily checked. In the first case, the character $\langle a+b-1, a+b-3, \ldots$, $b-a+1\rangle$ is not self-associate, and the associate character appears with the same multiplicity as $\mu$ is not a hook.

We now prove the statement of the Theorem by comparing degrees on both sides.

By the degree formulae for ordinary and spin characters we have for the left hand side:

$$
\langle n\rangle\left[b^{a}\right](1)=2^{\left[\frac{n-1}{2}\right]} \frac{n !}{H(a, b)},
$$

where we denote by $H(a, b)$ the product of the hook lengths in $\left(b^{a}\right)$. For the right hand side in the statement of the Theorem we obtain by the bar formula

$$
2^{\left[\frac{a-1}{2}\right]} \cdot 2^{\left[\frac{n-a}{2}\right]} \frac{n !}{B(a, b)}=2^{\left[\frac{n-1}{2}\right]} \frac{n !}{B(a, b)},
$$

where $B(a, b)$ denotes the product of the bar lengths in $(a+b-1, a+b-$ $3, \ldots, b-a+1)$.

Hence we have to prove that for all $a \leq b$ we have $H(a, b)=B(a, b)$.

For this, we divide the Young diagram resp. the shifted diagram into three regions:
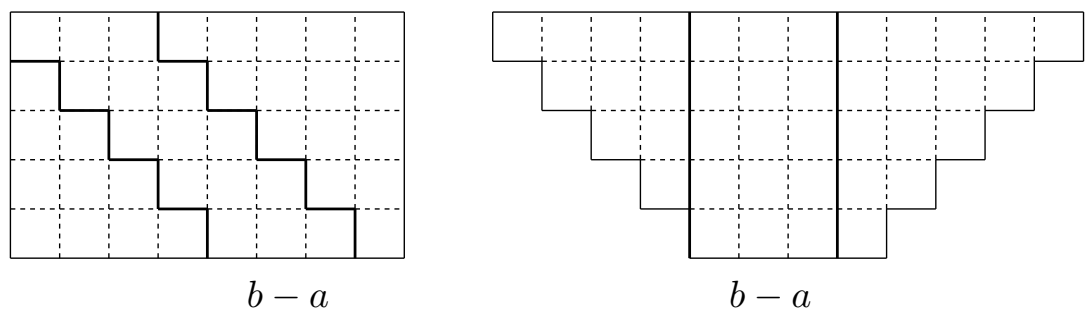

The middle region in both diagrams is of width $b-a$. It is easy to check that the hook lengths in the middle region of $\left(b^{a}\right)$ are exactly the same as the bar lengths in the middle region of $(a+b-1, a+b-3, \ldots, b-a+1)$; let 
$H_{m}(a, b)=B_{m}(a, b)$ denote the product of the hook lengths resp. bar lengths in these middle regions. Let $H_{l}(a, b)$ and $H_{r}(a, b)$ denote the product of the hook lengths in the left resp. right region of the diagram for $\left(b^{a}\right)$; similarly, let $B_{l}(a, b), B_{r}(a, b)$ denote the product of the bar lengths in the left resp. right region of the diagram for $(a+b-1, a+b-3, \ldots, b-a+1)$. The bar lengths occurring in the left region of $(a+b-1, a+b-3, \ldots, b-a+1)$ are exactly the same as the hook lengths in the left region of $\left(b^{a}\right)$ each multiplied by 2 , so

$$
B_{l}(a, b)=2^{\left(\begin{array}{c}
a \\
2
\end{array}\right)} H_{l}(a, b) .
$$

In the notation of Lemma 3.1 we have $H_{r}(a, b)=B(a)$ and $B_{r}(a, b)=H(a)$. Since by Lemma 3.1 we know that $B(a)=2^{\left(\begin{array}{l}a \\ 2\end{array}\right)} H(a)$, we obtain

$$
\begin{aligned}
H(a, b) & =H_{l}(a, b) H_{m}(a, b) H_{r}(a, b)=H_{l}(a, b) B_{m}(a, b) \cdot 2^{\left(\begin{array}{c}
a \\
2
\end{array}\right)} B_{r}(a, b) \\
& =B_{l}(a, b) B_{m}(a, b) B_{r}(a, b)=B(a, b)
\end{aligned}
$$

and thus the result is proved.

Next we deal with the natural character $[n-1,1]$ and describe some almost homogeneous products with this character.

Theorem 3.3. Let $n$ be a triangular number, say $n=\left(\begin{array}{c}k+1 \\ 2\end{array}\right)$. Then

$$
\langle k, k-1, \ldots, 2,1\rangle \cdot[n-1,1]=\langle k+1, k-\widehat{1, k-2}, \ldots, 3,2\rangle .
$$

Proof. First we check that the character given on the right hand side in the statement above does indeed appear as a constituent:

$$
\begin{aligned}
& (\langle k, k-1, \ldots, 2,1\rangle \cdot[n-1,1],\langle k+1, k-1, k-2, \ldots, 3,2\rangle) \\
= & (\langle k, k-1, \ldots, 2,1\rangle \cdot \overline{\langle k+1, k-1, k-2, \ldots, 3,2\rangle},[n-1,1]) \\
= & \left(\langle k, k-1, \ldots, 2,1\rangle \downarrow_{\widetilde{S}_{n-1}},\langle k+1, k-1, k-2, \ldots, 3,2\rangle \downarrow_{\widetilde{S}_{n-1}}\right) \\
= & 1
\end{aligned}
$$

where the last equality follows from the spin branching theorem. Now to prove the assertion it suffices to check degrees on both sides.

Let again denote $B(k)$ the product of the bar lengths in $(k, k-1, \ldots, 2,1)$, and let $B^{\prime}(k)$ denote the product of the bar lengths in $(k+1, k-1, k-2$, $\ldots, 2)$. Then by the bar formula we have to check whether the following equation holds:

$$
2^{\left[\frac{n-k}{2}\right]} \frac{n !}{B(k)}(n-1)=2^{\left[\frac{n-k+2}{2}\right]} \frac{n !}{B^{\prime}(k)}
$$

or equivalently,

$$
(n-1) B^{\prime}(k)=2 B(k) .
$$


We want to prove the claim by induction on $k$, starting with $k=3$, where the claim is easily checked. By Lemma 3.1

$$
B(k+1)=B(k) \prod_{j=k+1}^{2 k+1} j .
$$

Let $N=N(k-2)$ be the product of the bar lengths in $(k-1, k-2, \ldots, 2)$. Then by considering the shifted diagrams one sees that

$$
\begin{aligned}
B^{\prime}(k) & =N \cdot k(k+1) \prod_{j=k+3}^{2 k} j \\
B^{\prime}(k+1) & =N \cdot(k-1) k\left(\prod_{j=k+2}^{2 k-1} j\right)(k+1)(k+2) \prod_{i=k+4}^{2 k+2} i .
\end{aligned}
$$

Hence

$$
B^{\prime}(k+1)=B^{\prime}(k) \frac{(k-1)(k+2)(2 k+1)(2 k+2)}{k+3} \prod_{j=k+2}^{2 k-1} j .
$$

Since $n=\left(\begin{array}{c}k+1 \\ 2\end{array}\right)$, we know by induction that

$$
\left(k^{2}+k-2\right) B^{\prime}(k)=4 B(k),
$$

and we have to show that

$$
\left(k^{2}+3 k\right) B^{\prime}(k+1)=4 B(k+1) .
$$

Using the relations given above this is a straightforward calculation.

Hence the assertion of the Theorem is proved.

Theorem 3.4. Let $n \in \mathbb{N}, n \geq 3$, and let $\mu \in D(n)$. Then the product $\langle\mu\rangle \cdot[n-1,1]$ is irreducible if and only if $n$ is a triangular number, say $n=\left(\begin{array}{c}k+1 \\ 2\end{array}\right)$, with $k \equiv 2$ or $3 \bmod 4$, and $\mu=(k, k-1, \ldots, 2,1)$. In this case,

$$
\langle k, k-1, \ldots, 2,1\rangle \cdot[n-1,1]=\langle k+1, k-1, k-2, \ldots, 3,2\rangle .
$$

Proof. If $\mu$ and $k$ are as stated, then $\mu$ is odd and $(k+1, k-1, k-2, \ldots, 3,2)$ is even, and so by the previous Theorem the stated product is indeed irreducible.

Now assume that the product $\langle\mu\rangle \cdot[n-1,1]$ is irreducible. By the classification result for products with the basic spin character, we know that $\mu \neq(n)$.

So assume now $\mu \neq(k, k-1, \ldots, 1)$. Then by Theorem 2.5 resp. by Theorem 2.4 both $[n-2,2]$ and $[n]$ are constituents of the product $\langle\mu\rangle \cdot \overline{\langle\mu\rangle}$ as well as of the square $[n-1,1]^{2}$. Hence

$$
([n-1,1] \cdot\langle\mu\rangle,[n-1,1] \cdot\langle\mu\rangle)=(\langle\mu\rangle \cdot \overline{\langle\mu\rangle},[n-1,1] \cdot[n-1,1]) \geq 2,
$$

so the product is not irreducible. 
For the classification of the irreducible mixed products, we will need some further information in the special case of staircase partitions.

Theorem 3.5. Let $k \in \mathbb{N}, n=\left(\begin{array}{c}k+1 \\ 2\end{array}\right)$. Define the coefficients $a_{1}, a_{2}, a_{3}, b_{2}$, $b_{3}, c_{3}$ by

$$
\begin{aligned}
\langle k, \ldots, 1\rangle \cdot \overline{\langle k, \ldots, 1\rangle}= & {[n]+a_{1}[n-1,1]+a_{2}[n-2,2]+a_{3}[n-3,3] } \\
& +b_{2}\left[n-2,1^{2}\right]+b_{3}\left[n-3,1^{3}\right]+c_{3}[n-3,2,1] \\
& + \text { other constituents. }
\end{aligned}
$$

Then:

(i) $a_{1}=a_{2}=c_{3}=0$.

(ii) $b_{2}= \begin{cases}1 & \text { if } k \equiv 0 \text { or } 1 \bmod 4 \\ 0 & \text { if } k \equiv 2 \text { or } 3 \bmod 4 .\end{cases}$

(iii) $a_{3}=b_{3}=1$.

Proof. Set $\rho_{k}=(k, \ldots, 1)$ and $\varphi_{k}=\langle k, \ldots, 1\rangle$. Note that $\rho_{k} \in D^{+}$if and only if $k \equiv 0$ or $1 \bmod 4$. Also, let $\pi_{\alpha}=\mathbf{1}_{S_{\alpha}} \uparrow^{S_{n}}=\mathbf{1}_{\widetilde{S}_{\alpha}} \uparrow^{\widetilde{S}_{n}}$. So

$$
\left(\varphi_{k} \cdot \overline{\varphi_{k}}, \pi_{\alpha}\right)=\left(\varphi_{k} \downarrow_{\widetilde{S}_{\alpha}}, \varphi_{k} \downarrow_{\widetilde{S}_{\alpha}}\right),
$$

and for computing the restriction we use the spin branching theorem resp. the shifted Littlewood-Richardson Rule provided by Theorem 2.1.

Since $\varphi_{k} \downarrow_{\widetilde{S}_{(n-1,1)}}=\langle k, \ldots, 2\rangle,\left(\varphi_{k} \cdot \overline{\varphi_{k}}, \pi_{(n-1,1)}\right)=1$. As $[n-1,1]=$ $\pi_{(n-1,1)}-[n]$, this yields

$$
a_{1}=\left(\varphi_{k} \cdot \overline{\varphi_{k}},[n-1,1]\right)=0 .
$$

Next, $\varphi_{k} \downarrow_{\widetilde{S}_{(n-2,2)}}$ is the irreducible character $\langle k, \ldots, 3,1\rangle \times_{c}\langle 2\rangle$ (up to the choice of associates in the case $k \equiv 0$ or $1 \bmod 4)$, hence $\left(\varphi_{k} \cdot \overline{\varphi_{k}}, \pi_{(n-2,2)}\right)=1$. As $[n-2,2]=\pi_{(n-2,2)}-\pi_{(n-1,1)}$, this implies

$$
a_{2}=\left(\varphi_{k} \cdot \overline{\varphi_{k}},[n-2,2]\right)=0 .
$$

The restriction $\varphi_{k} \downarrow_{\widetilde{S}_{(n-3,3)}}$ has the two irreducible constituents $\langle k, \ldots, 4$, $2,1\rangle \times_{c}\langle 3\rangle$ and $\langle k, \ldots, 3\rangle \times_{c}\langle 2,1\rangle$ (up to the choice of associates in the case $k \equiv 2$ or $3 \bmod 4)$. Hence $\left(\varphi_{k} \cdot \overline{\varphi_{k}}, \pi_{(n-3,3)}\right)=2$, and from the equation $[n-3,3]=\pi_{(n-3,3)}-\pi_{(n-2,2)}$ we now deduce

$$
a_{3}=\left(\varphi_{k} \cdot \overline{\varphi_{k}},[n-3,3]\right)=1 .
$$

Now $\varphi_{k} \downarrow_{\widetilde{S}_{\left(n-2,1^{2}\right)}}=\langle k, \overline{\ldots, 3}, 1\rangle$. Thus,

$$
\left(\varphi_{k} \cdot \overline{\varphi_{k}}, \pi_{\left(n-2,1^{2}\right)}\right)= \begin{cases}1 & \text { if } k \equiv 2 \text { or } 3 \bmod 4 \\ 2 & \text { if } k \equiv 0 \text { or } 1 \bmod 4 .\end{cases}
$$


As $\left[n-2,1^{2}\right]=\pi_{\left(n-2,1^{2}\right)}-\pi_{(n-2,2)}-[n-1,1]$, we obtain

$$
b_{2}=\left(\varphi_{k} \cdot \overline{\varphi_{k}},\left[n-2,1^{2}\right]\right)= \begin{cases}0 & \text { if } k \equiv 2 \text { or } 3 \bmod 4 \\ 1 & \text { if } k \equiv 0 \text { or } 1 \bmod 4 .\end{cases}
$$

For the restriction $\varphi_{k} \downarrow_{\widetilde{S}_{(n-3,2,1)}}$ we obtain

$$
\langle k, \ldots, \widehat{4,2,1}\rangle \times_{c}\langle 2\rangle+\langle k, \ldots, 4,3\rangle \times_{c}\langle 2\rangle
$$

if $k \equiv 0$ or $1 \bmod 4$, and

$$
\langle k, \ldots, 4,2,1\rangle \times_{c}\langle 2\rangle+\langle k, \ldots, 4,3\rangle \times_{c}\langle 2\rangle
$$

up to a choice of associates for the second summand, if $k \equiv 2$ or $3 \bmod 4$. Hence

$$
\left(\varphi_{k} \cdot \bar{\varphi}_{k}, \pi_{(n-3,2,1)}\right)= \begin{cases}3 & \text { if } k \equiv 0 \text { or } 1 \bmod 4 \\ 2 & \text { if } k \equiv 2 \operatorname{or} 3 \bmod 4\end{cases}
$$

As

$$
[n-3,2,1]=\frac{1}{2}\left(\pi_{(n-3,2,1)}-\pi_{(n-3,3)}-[n-2,2]-[n-1,1]-\left[n-2,1^{2}\right]\right),
$$

we obtain

$$
c_{3}=\left(\varphi_{k} \cdot \overline{\varphi_{k}},[n-3,2,1]\right)=0 .
$$

Finally, we have

$$
\varphi_{k} \downarrow_{\widetilde{S}_{\left(n-3,1^{3}\right)}}= \begin{cases}\langle\hat{, \ldots, 3\rangle}+2\langle k, \ldots, 4,2,1\rangle & \text { if } k \equiv 0 \text { or } 1 \bmod 4 \\ \langle k, \ldots, 3\rangle+\langle k, \ldots, 4,2,1\rangle & \text { if } k \equiv 2 \text { or } 3 \bmod 4 .\end{cases}
$$

Thus

$$
\left(\varphi_{k} \cdot \overline{\varphi_{k}}, \pi_{\left(n-3,1^{3}\right)}\right)= \begin{cases}6 & \text { if } k \equiv 0 \text { or } 1 \bmod 4 \\ 3 & \text { if } k \equiv 2 \text { or } 3 \bmod 4 .\end{cases}
$$

As

$$
\left[n-3,1^{3}\right]=\pi_{\left(n-3,1^{3}\right)}-\pi_{(n-3,2,1)}-[n-1,1]-[n-2,2]-2\left[n-2,1^{2}\right],
$$

we obtain

$$
b_{3}=\left(\varphi_{k} \cdot \overline{\varphi_{k}},\left[n-3,1^{3}\right]\right)=1 \text {. }
$$

Theorem 3.6. Let $\lambda \in P(n), \lambda \neq(n),\left(1^{n}\right)$. Let $\mu \in D(n)$. Then $[\lambda] \cdot\langle\mu\rangle$ is irreducible if and only if one of the following occurs:

(i) $n=2 k, \lambda=(k, k)$ or $\left(2^{k}\right)$ and $\mu=(n)$.

Here the products are

$$
[k, k] \cdot\langle n\rangle=\left[2^{k}\right] \cdot\langle n\rangle=\langle k+1, k-1\rangle .
$$

(ii) $n=\left(\begin{array}{c}k+1 \\ 2\end{array}\right)$ for some $k \in \mathbb{N}$ with $k \equiv 2$ or $3 \bmod 4, \lambda=(n-1,1)$ or $\left(2,1^{n-2}\right)$ and $\mu=(k, k-1, \ldots, 2,1)$.

Here the products are

$$
[n-1,1] \cdot\langle k, \ldots, 1\rangle=\left[2,1^{n-2}\right] \cdot\langle k, \ldots, 1\rangle=\langle k+1, k-1, k-2, \ldots, 2\rangle .
$$


Proof. In the cases (i) and (ii) described above, the product is irreducible by Theorem 3.2 resp. Theorem 3.4. In the following we may assume that $n \geq 4$.

Now assume that $[\lambda] \cdot\langle\mu\rangle$ is irreducible. Then

$$
1=([\lambda] \cdot\langle\mu\rangle,[\lambda] \cdot\langle\mu\rangle)=\left([\lambda]^{2},\langle\mu\rangle \cdot \overline{\langle\mu\rangle}\right) .
$$

As both $[\lambda]^{2}$ and $\langle\mu\rangle \cdot \overline{\langle\mu\rangle}$ have $[n]$ as a constituent, it suffices to find a further common constituent in all situations not covered by (i) and (ii).

By Theorem 2.4, $[\lambda]^{2}$ always contains a constituent $[n-2,2]$, and by Theorem 2.5, also $\langle\mu\rangle \cdot \overline{\langle\mu\rangle}$ contains a constituent $[n-2,2]$, unless $\mu=(n)$ or $\mu=(k, k-1, \ldots, 1)$. So we only have to consider mixed products $[\lambda] \cdot\langle\mu\rangle$ with $\mu$ of these two exceptional types.

For $\mu=(n)$, this was done in Theorem 3.2, giving the products described in case (i) as the only irreducible mixed products with the basic spin character.

So it remains to deal with the case of a staircase $\mu=(k, k-1, \ldots, 1)$. By Theorem 2.4 and Theorem 3.5 we then find a common constituent $\left[n-3,1^{3}\right]$ in $[\lambda]^{2}$ and $\langle\mu\rangle \cdot \overline{\langle\mu\rangle}$, unless $\lambda$ is $(n-1,1)$ or $(n-1,1)^{\prime}$. But in this latter situation, we can apply Theorem 3.3 which leads exactly to the irreducible mixed products given in (ii).

Acknowledgements. The financial support by the Deutsche Forschungsgemeinschaft (grants Be 923/6-1 and JAP-115/169/0) at different stages of the work for this article is gratefully acknowledged. Special thanks for the hospitality enjoyed at the University of Osaka where some part of the work for this article was done go to Professor Uno. The author is also grateful to John Stembridge for sharing his maple packages SF and QF, that helped finding homogeneous mixed products.

\section{References}

[1] C. Bessenrodt and A. Kleshchev, On Kronecker products of complex representations of the symmetric groups, Pacific J. Math., 190(2) (1999), 201-223, MR 2000i:20017.

[2] C. Bessenrodt and A. Kleshchev, On Kronecker products of spin characters of the double covers of the symmetric groups, Pacific J. Math., 198 (2001), 295-305.

[3] M. Clausen and H. Meier, Extreme irreduzible Konstituenten in Tensordarstellungen symmetrischer Gruppen, Bayreuther Math. Schriften, 45 (1993), 1-17, MR 94j:20009, Zbl 799.05004.

[4] Y. Dvir, On the Kronecker product of $S_{n}$ characters, J. Algebra, 154 (1993), 125-140, MR 94a:20023, Zbl 848.20006.

[5] P. Hoffman and J.F. Humphreys, Projective Representations of the Symmetric Groups, Oxford, 1992, MR 94f:20027, Zbl 777.20005.

[6] G. James, The Representation Theory of the Symmetric Groups, Springer Lecture Notes Math., 682 (1978), MR 80g:20019, Zbl 393.20009. 
[7] G. James and A. Kerber, The Representation Theory of the Symmetric Group, Addison-Wesley, London, 1981, MR 83k:20003, Zbl 491.20010.

[8] P.B. Kleidman and D.B. Wales, The projective characters of the symmetric groups that remain irreducible on subgroups, J. Algebra, 138 (1991), 440-478, MR 92e:20008, Zbl 792.20012.

[9] I.G. Macdonald, Symmetric Functions and Hall Polynomials, 2nd edition, Oxford Univ. Press, Oxford, 1995, MR 96h:05207, Zbl 824.05054.

[10] A.O. Morris, The spin representation of the symmetric group, Proc. London Math. Soc., 12(3) (1962), 55-76, MR 25 \#133, Zbl 104.25202.

[11] A.O. Morris, The spin representation of the symmetric group, Canad. J. Math., 17 (1965), 543-549, MR 31 \#240, Zbl 135.05602.

[12] J. Saxl, The complex characters of the symmetric groups that remain irreducible in subgroups, J. Algebra, 111 (1987), 210-219, MR 88i:20011, Zbl 633.20008.

[13] J. Stembridge, Shifted tableaux and the projective representations of symmetric groups, Adv. Math., 74 (1989), 87-134, MR 90k:20026, Zbl 677.20012.

[14] E. Vallejo, On the Kronecker product of irreducible characters of the symmetric group, preprint.

[15] I. Zisser, The character covering numbers of the alternating groups, J. Algebra, 153 (1992), 357-372, MR 94b:20021, Zbl 789.20013.

Received September 9, 1999.

FAKULtät FÜR MATHEMATIK

Otto-von-Guericke-Universität Magdeburg

D-39016 Magdeburg

Germany

E-mail address: bessen@mathematik.uni-magdeburg.de 